\title{
ARTICLE
}

\section{Interfacial superconductivity in a bi-collinear antiferromagnetically ordered FeTe monolayer on a topological insulator}

S. Manna ${ }^{1, *}$, A. Kamlapure ${ }^{1, \star}$, L. Cornils ${ }^{1, \star}$, T. Hänke${ }^{1}$, E.M.J. Hedegaard ${ }^{2}$, M. Bremholm², B.B. Iversen ${ }^{2}$, Ph. Hofmann ${ }^{3}$, J. Wiebe ${ }^{1} \&$ R. Wiesendanger ${ }^{1}$

The discovery of high-temperature superconductivity in Fe-based compounds triggered numerous investigations on the interplay between superconductivity and magnetism, and on the enhancement of transition temperatures through interface effects. It is widely believed that the emergence of optimal superconductivity is intimately linked to the suppression of long-range antiferromagnetic (AFM) order, although the exact microscopic picture remains elusive because of the lack of atomically resolved data. Here we present spinpolarized scanning tunnelling spectroscopy of ultrathin $\mathrm{FeTe}_{1-x} \mathrm{Se}_{x}(x=0,0.5)$ films on bulk topological insulators. Surprisingly, we find an energy gap at the Fermi level, indicating superconducting correlations up to $T_{\mathrm{c}} \sim 6 \mathrm{~K}$ for one unit cell FeTe grown on $\mathrm{Bi}_{2} \mathrm{Te}_{3}$, in contrast to the non-superconducting bulk FeTe. The gap spatially coexists with bi-collinear AFM order. This finding opens perspectives for theoretical studies of competing orders in Fe-based superconductors and for experimental investigations of exotic phases in superconducting layers on topological insulators.

\footnotetext{
${ }^{1}$ Department of Physics, University of Hamburg, Jungiusstrasse 11, D-20355 Hamburg, Germany. ${ }^{2}$ Department of Chemistry and iNANO, Center for Materials Crystallography, Aarhus University, Langelandsgade 140, DK-8000 Aarhus C, Denmark. ${ }^{3}$ Department of Physics and Astronomy, Interdisciplinary Nanoscience Center, Aarhus University, DK-8000 Aarhus C, Denmark. *These authors contributed equally to this work. Correspondence and requests for materials should be addressed to S.M. (email: smanna@physnet.uni-hamburg.de) or to J.W. (email: jwiebe@physnet.uni-hamburg.de).
} 
$\mathrm{T}$ opological insulators (TIs) and interfacial superconductors (SCs) are both topics of intense current interest in modern condensed matter physics ${ }^{1,2}$. The combination of both materials is expected to reveal novel physics such as, for example, Majorana Fermions arising in heterostructures of TIs and s-wave SCs by including magnetic fields ${ }^{3}$. Most experimental studies of such heterostructures have concentrated on TI films grown on superconducting bulk substrates ${ }^{4}$. Alternatively, SC/TI heterostructures could be realized by growing superconducting films on top of pristine bulk TIs.

In the past years, studies of Fe-based SCs and their electronic and magnetic properties have been one of the major research fields in condensed matter physics ${ }^{5,6}$. The recent finding that the superconducting transition temperature $T_{\mathrm{c}}$ can be pushed above $100 \mathrm{~K}$ in an FeSe unit cell (UC) thin film grown epitaxially on $\mathrm{SrTiO}_{3}$ (refs 7-9) spurred numerous investigations aiming at an exact understanding of how the superconductivity in transition metal mono- and dichalcogenides evolves from bulk to ultrathin films. Bulk FeSe crystals exhibit a $T_{\mathrm{c}}$ of no higher than $8 \mathrm{~K}$ (ref. 10). The significant interfacial enhancement of $T_{\mathrm{c}}$ in UC FeSe on $\mathrm{SrTiO}_{3}$ as well as in similar systems, such as FeSe on $\mathrm{BaTiO}_{3}$ (ref. 11) and $\mathrm{FeTe}_{1-x} \mathrm{Se}_{x}$ on $\mathrm{SrTiO}_{3}{ }^{12}$, leads to a revival of the idea that tailoring the electron pairing by interfacing a SC with another material can be used to achieve high-temperature $\mathrm{SC}^{1}$. Interestingly, $\mathrm{SC}$ can even emerge by interfacing two insulators ${ }^{13}$ or a $\mathrm{TI}\left(\mathrm{Bi}_{2} \mathrm{Te}_{3}\right)$ with a non-SC Fe-chalcogenide $(\mathrm{FeTe})^{14,15}$. It is therefore of great importance to understand how different substrates affect the electronic, spin-dependent and superconducting properties of single-layer Fe-based SCs. The latter studies ${ }^{14,15}$ raised the fundamental question of what happens to the bi-collinear antiferromagnetic (AFM) structure of FeTe (ref. 16) once an interfacial SC state is established in contact with $\mathrm{Bi}_{2} \mathrm{Te}_{3}$. In the work of $\mathrm{He}$ et al. ${ }^{14}$, it was proposed that the topological surface state may dope the FeTe and suppress the long-range AFM order at the interface, thereby inducing the observed interfacial SC.
By a low-temperature spin-polarized scanning tunnelling spectroscopy (SP-STS) investigation of the correlation between the film structure, the local electronic, superconducting and spindependent properties, we show here that, in contrast to this assumption, the energy gap at the Fermi level in UC thin FeTe films epitaxially grown on $\mathrm{Bi}_{2} \mathrm{Te}_{3}(111)$ can spatially coexist on the atomic scale with long-range bi-collinear AFM order.

\section{Results}

Structural properties of $\mathrm{FeTe}_{1-x} \mathrm{Se}_{x}$ thin films. We have prepared ultrathin epitaxial $\mathrm{FeTe}$ films on $\mathrm{Bi}_{2} \mathrm{Te}_{3}$ single crystals by in situ deposition of $\mathrm{Fe}$ on the TI substrates under ultrahigh vacuum (UHV) conditions, followed by an in situ annealing treatment (see Methods section). Figure 1a depicts a constantcurrent scanning tunnelling microscopy (STM) topographic image of such a film with FeTe islands of different thicknesses, together with regions of the bare substrate. We distinguish between these different areas based on the measured step heights and differences in the observed atomic-scale surface structures. The line profile displayed in Fig. $1 \mathrm{~b}$ taken across three different terraces separated by two steps reveals a step height $h(\mathrm{a}-\mathrm{b})$ of $h \sim 0.65 \pm 0.05 \mathrm{~nm}$, roughly equal to the $c$ axis lattice constant $(0.62 \mathrm{~nm})$ of bulk $\mathrm{FeTe}$, while the right step $b-c$ is considerably smaller $(h \sim 0.35 \pm 0.05 \mathrm{~nm})$. The smaller step results from an FeTe UC being embedded in the topmost quintuple layer of the substrate, most probably as sketched in Fig. 1b. Figure le presents an atomically resolved STM topographic image of the substrate area $\left(\mathrm{Bi}_{2} \mathrm{Te}_{3}\right)$, which reveals the hexagonal close-packed atomic lattice (see inset Fast Fourier transform (FFT) image) of top Te atoms with a spacing of $\sim 0.44 \mathrm{~nm}$. A topographic STM image of the top UC layer of $\mathrm{FeTe}$, with an atomic contrast determined by the topmost chalcogenide (Te) layer (Fig. 1f), reveals that the $a$ axis of the FeTe is aligned parallel to one of the closed packed directions of the substrate Te atoms. The period of the atomic lattice along the $a$ axis direction is $\sim 0.38 \mathrm{~nm}$, which is consistent
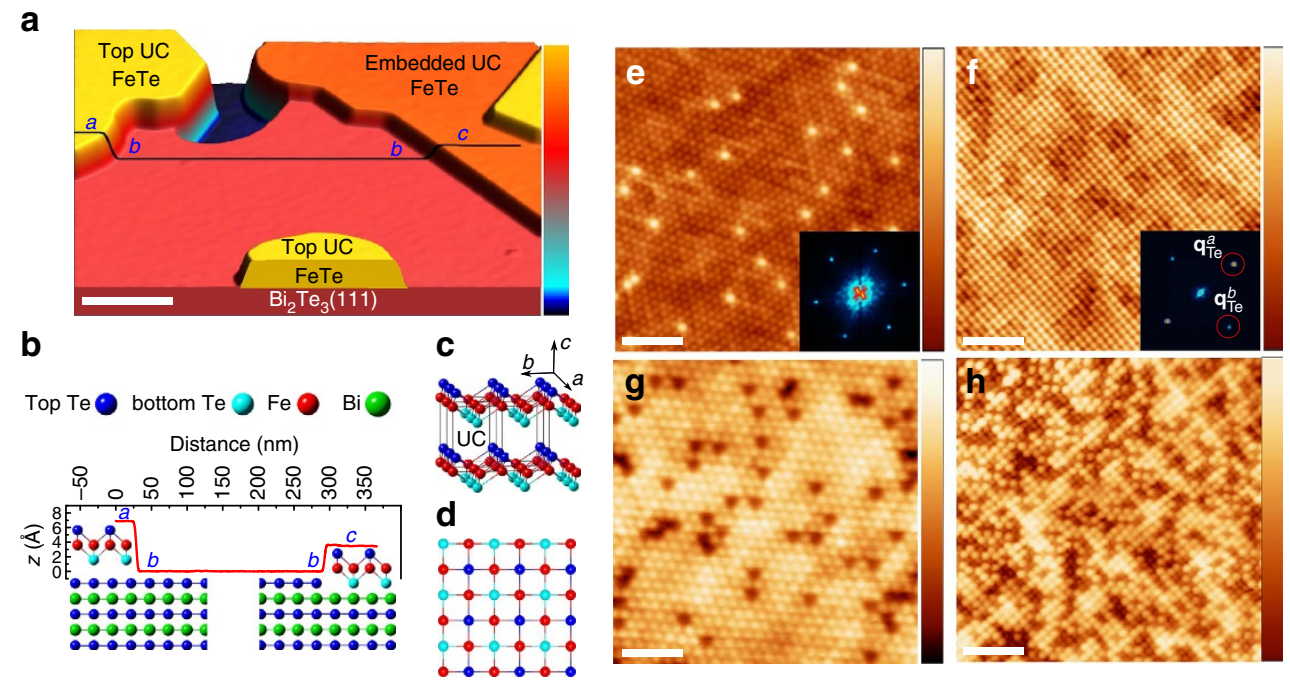

Figure 1 | STM topography of $\mathbf{F e T e}_{\mathbf{1}_{\boldsymbol{x}}} \mathbf{S e}_{\boldsymbol{x}}$ ultrathin films. (a) Large-scale constant-current image $\left(U=500 \mathrm{mV}, I_{\mathrm{s}}=20 \mathrm{pA}\right.$, white scale bar, $80 \mathrm{~nm}$ wide, colour scale from 0 to $1.58 \mathrm{~nm}$ apparent height) of one UC FeTe grown on a $\mathrm{Bi}_{2} \mathrm{Te}_{3}$ substrate. (b) Measured height profile along the black line ( $a-b-c$ ) in a revealing embedded $(h=0.35 \mathrm{~nm})$ and top UC $(h=0.68 \mathrm{~nm})$ FeTe, consistent with the suggested cross-sectional schematic illustration of the $\mathrm{FeTe} / \mathrm{Bi}_{2} \mathrm{Te}_{3}$ heterostructure (not to scale). The schematic crystal structure of an FeTe UC is additionally shown in side- (c) and top view (d). (e) Atomically resolved STM images of $\mathrm{Bi}_{2} \mathrm{Te}_{3}$ substrate region $\left(U=-200 \mathrm{mV}, I_{\mathrm{s}}=100 \mathrm{pA}, 3 \mathrm{~nm}\right.$ lateral scale bar, colour scale from 0 to $\left.105 \mathrm{pm}\right)$, and (f) of a top $U \mathrm{C}$ thin FeTe layer $\left(U=140 \mathrm{mV}, I_{\mathrm{s}}=100 \mathrm{pA}, 3 \mathrm{~nm}\right.$ lateral scale bar, colour scale from 0 to $84 \mathrm{pm}$ ). FFTs (image sizes $0.75 \AA^{-1}$ ) of the images are displayed in the insets of $\mathbf{e}, \mathbf{f}$, revealing the Bragg peaks associated with the top Te atomic lattice at $\mathbf{q}_{\mathrm{Te}}^{a}$ and $\mathbf{q}_{\mathrm{Te}}^{b}$. $(\mathbf{g})$ Atomically resolved $\mathrm{STM}_{\mathrm{Tmages}}$ of the $\mathrm{Bi}_{2} \mathrm{Te}_{1.8} \mathrm{Se}_{1.2}$ substrate $\left(U=50 \mathrm{mV}, I_{\mathrm{s}}=1 \mathrm{nA}, 2 \mathrm{~nm}\right.$ lateral scale bar, colour scale from 0 to $\left.49 \mathrm{pm}\right)$ and $(\mathbf{h})$ of one $U C$ FeTe $\mathrm{H}_{0.5} \mathrm{Se}_{0.5}$ layer grown on top $(U=100 \mathrm{mV}$, $I_{\mathrm{s}}=740 \mathrm{pA}, 3 \mathrm{~nm}$ lateral scale bar, colour scale from 0 to $159 \mathrm{pm}$ ). 
with the Te-Te atomic distance on the surface of bulk FeTe (ref. 17). The period along the $b$ axis is slightly smaller ( $a$ / $b \sim 1.04$ ), which might be partly attributed to the transition into the orthorhombic/monoclinic phase, known for bulk $\mathrm{FeTe}^{18}$, but could be enhanced by uniaxial strain as a result of the heteroepitaxial growth. This holds for the embedded UC FeTe as well as for thicker layers (Supplementary Fig. 1). However, there is no overall periodic height modulation of the UC FeTe layers, and only in some areas of the thinnest islands irregular wrinkles are observed. This indicates a rather weak interaction of the FeTe layer with the substrate, in contrast to what has been observed for FeSe grown on $\mathrm{Bi}_{2} \mathrm{Se}_{3}$ (ref. 19). The Fourier transform of the topographic image (inset of Fig. 1f) clearly shows the peaks associated with the almost square Te atomic lattice, with the spots along the $a$ direction $\left(\mathbf{q}_{T e}^{a}\right)$ slightly more intense than those along the $b$ direction ( $\mathbf{q}_{\mathrm{Te}}^{b}$; ref. 16). Despite the different lattice symmetries (sixfold for $\mathrm{Bi}_{2} \mathrm{Te}_{3}$ and fourfold for $\mathrm{FeTe}$ ) with respect to the $c$ axis directions and the relatively large lattice mismatch, the heteroepitaxial growth is of very high quality, resulting in an atomically sharp and defect-free interface. In contrast to the surface of bulk FeTe (ref. 16), the surfaces of our ultrathin FeTe films do not show any excess $\mathrm{Fe}$ atoms, indicating a stoichiometric FeTe layer.

Although the focus of the present study is on the single layer of FeTe on $\mathrm{Bi}_{2} \mathrm{Te}_{3}(111)$, ultrathin $\mathrm{FeTe}_{1-x} \mathrm{Se}_{x}(x=0.5)$ films grown on the ternary $\mathrm{TI} \mathrm{Bi}_{2} \mathrm{Te}_{1.8} \mathrm{Se}_{1.2}(111)$ serve as an important reference system ${ }^{20}$. In the corresponding bulk system, FeTe exhibits a bi-collinear AFM order, while superconductivity can be induced and substantially enhanced up to $15 \mathrm{~K}$ with partial substitution of Se for Te at $x=0.5$ (ref. 12). It is therefore particularly interesting to investigate how the superconducting state develops from a magnetically ordered state with Se substitution in the thin-film system we studied here.

High-quality, one UC thin $\mathrm{FeTe}_{0.5} \mathrm{Se}_{0.5}$ films (Supplementary Fig. 2) with well-defined stoichiometry were epitaxially grown on $\mathrm{Bi}_{2} \mathrm{Te}_{1.8} \mathrm{Se}_{1.2}(111)$ substrates using a similar preparation method as for the FeTe films. Atomically resolved topographic STM images of the cleaved $\mathrm{Bi}_{2} \mathrm{Te}_{1.8} \mathrm{Se}_{1.2}$ substrate surface (Fig. $1 \mathrm{~g}$ ) show $\mathrm{Se}$ and $\mathrm{Te}$ atoms in the topmost layer of the surface appearing with slightly darker and brighter contrast, respectively, with a frequency in accordance with the stoichiometry ${ }^{21}$. A typical topographic STM image of a one UC thin $\mathrm{FeTe}_{0.5} \mathrm{Se}_{0.5}$ film grown on top (Fig. 1h) reveals an ordered square lattice of Se/Te atoms with about half of the atoms appearing brighter (52\%, Te sites) while the other half appears darker ( $48 \%$, Se sites), with a height difference of $65 \pm 15 \mathrm{pm}$, close to the value found for similarly doped $\mathrm{FeTe}_{1-x} \mathrm{Se}_{x}$ samples ${ }^{12,22}$.

Characterization of superconducting properties. To characterize the electronic structure of these films, STS measurements of the differential tunnelling conductance $(\mathrm{d} I / \mathrm{d} U)$ as a function of the applied bias voltage were performed, reflecting the sample's local density of states. Figure 2a shows single tunnelling spectra measured on the one UC thin $\mathrm{FeTe}_{0.5} \mathrm{Se}_{0.5}$ film and on the $\mathrm{Bi}_{2} \mathrm{Te}_{1.8} \mathrm{Se}_{1.2}$ substrate at a temperature of $T=1.1 \mathrm{~K}$. The $\mathrm{FeTe}_{0.5} \mathrm{Se}_{0.5}$ film exhibits an overall U-shaped $\mathrm{d} \mathrm{I} / \mathrm{d} U$ spectrum with an almost vanishing conductance value at the Fermi level $\left(E_{\mathrm{F}}\right)$ and an energy gap of $\Delta=2.5 \mathrm{meV}$, defined by half the distance between the two sharp coherence peaks in Fig. 2a. This measured gap value is of the same order of magnitude as measured on the surface of the corresponding superconducting bulk material ${ }^{23}$. In order to determine the critical temperature $T_{\mathrm{c}}$ of our $\mathrm{FeTe}_{0.5} \mathrm{Se}_{0.5}$ films, we performed temperature-dependent measurements of $\mathrm{d} I / \mathrm{d} U$ from 1.1 to $11 \mathrm{~K}$ (see Fig. 2b). As expected, the observed energy gap in $\mathrm{d} I / \mathrm{d} U$ becomes shallower with increasing temperature and eventually disappears at $\sim 11 \mathrm{~K}$. The energy gap values for different temperatures (shown in Fig. 2c) were extracted from fits of the background-corrected and symmetrized $\mathrm{d} I / \mathrm{d} U$ spectra (for data processing see Supplementary Fig. 3) employing the Dynes density of states, $N(E)=N_{\mathrm{n}}\left(E_{\mathrm{F}}\right) \cdot \Re\left[\frac{|E|-i \Gamma}{\sqrt{(|E|-i \Gamma)^{2}-\Delta^{2}(T)}}\right]$, with the density of states in the normal state at the Fermi energy $N_{\mathrm{n}}\left(E_{\mathrm{F}}\right)$ and the real part $\Re$, where the parameter $\Gamma$ accounts for the lifetime broadening of the quasiparticles ${ }^{24}$. The fitting of the temperature dependence of the gap in the framework of the Bardeen-Cooper-Schrieffer (BCS) theory ${ }^{25}$ yields an energy gap at $0 \mathrm{~K}$ of $\Delta_{0}=2.0 \mathrm{meV}$ and an extracted $T_{\mathrm{c}}=11 \mathrm{~K}$, similar to the values that have been extracted from STS on the corresponding superconducting bulk materials ${ }^{23,26}$. The temperature dependence of the $\mathrm{d} I / \mathrm{d} U$ spectra as well as the derived $T_{\mathrm{c}}$ value provide strong evidence that the observed gap feature arises from superconductivity in the ultrathin $\mathrm{FeTe}_{0.5} \mathrm{Se}_{0.5}$ film grown on $\mathrm{Bi}_{2} \mathrm{Te}_{1.8} \mathrm{Se}_{1.2}$.

Now we turn to the investigation of the electronic and magnetic structure of ultrathin $\mathrm{FeTe}$ films grown on $\mathrm{Bi}_{2} \mathrm{Te}_{3}$. Figure $2 \mathrm{~d}$ shows a series of spatially averaged $\mathrm{d} I / \mathrm{d} U$ spectra measured at $T=1.1 \mathrm{~K}$ on the $\mathrm{Bi}_{2} \mathrm{Te}_{3}$ substrate as well as on ultrathin FeTe films of different thicknesses. For both embedded UC and top UC FeTe layers a reduced $\mathrm{d} I / \mathrm{d} U$ at $E_{\mathrm{F}}$ with diffuse peaks at energies of \pm 1 to $\pm 2 \mathrm{meV}$ are found with a gap width and occurrence of the peaks varying with position (Supplementary Fig. 4). A gap of this size and temperature dependence (shown below) has not been observed at the surface of bulk FeTe (refs 27,28) or for FeTe single layers grown on $\mathrm{SrTiO}_{3}$ (ref. 12). The Fermi-level gap gradually vanishes for increased thicknesses of $\mathrm{FeTe}$ layers on $\mathrm{Bi}_{2} \mathrm{Te}_{3}$. For example, two UC thin FeTe films show a gap with a significantly reduced depth at $E_{\mathrm{F}}$. This behaviour suggests that the $\mathrm{FeTe} / \mathrm{Bi}_{2} \mathrm{Te}_{3}$ interface plays a significant role for the observed gap structure. In order to reveal the origin of the energy gap in one UC thin FeTe films grown on $\mathrm{Bi}_{2} \mathrm{Te}_{3}$, we thoroughly investigated the temperature and field dependence of the $d I / d U$ spectra. Figure $2 \mathrm{e}$ shows a series of background-corrected and symmetrized temperature-dependent $\mathrm{d} / \mathrm{d} U$ spectra for top $\mathrm{UC} F e T e$ on $\mathrm{Bi}_{2} \mathrm{Te}_{3}$. The tunnelling spectrum at $T=1.1 \mathrm{~K}$ exhibits a pair of diffuse peaks at $U \sim 2 \mathrm{meV}$ together with a gap region of reduced local density of states at $E_{\mathrm{F}}$. We observe that the gap depth and peak height in $\mathrm{d} I / \mathrm{d} U$ decrease with increasing temperature and finally vanish above $T=6 \mathrm{~K}$, very reminiscent of a superconducting transition. In order to test this hypothesis, the temperature-dependent spectra were fitted to a Dynes density of states (Fig. 2e). Here we note that a considerable lifetime broadening $\Gamma$ had to be assumed (Supplementary Fig. 5). The temperature dependence of the fitted energy gap values (Fig. 2f) resembles that of a superconducting phase transition ( $c f$. Fig. 2c). Kondo correlations, which were shown to cause dip features at $E_{\mathrm{F}}$ for heavy Fermion systems ${ }^{29}$ and might be present on the Fe lattice as well, are ruled out, as they would show a different temperature dependency of the gap depth (Supplementary Fig. 6). In addition, unlike under-doped cuprates, where a pseudogap phase appears above $T_{\mathrm{c}}($ ref. 30$)$, the gap in our tunnelling spectroscopy data always closes at $T_{\mathrm{c}} \sim 6 \mathrm{~K}$, thereby ruling out the presence of a pseudogap state. The spectral features therefore strongly suggest superconducting correlations of the UC FeTe layer. In order to extract the corresponding $T_{\mathrm{c}}$, we fit the temperature dependence of the gap in the framework of the BCS theory (Fig. 2f), resulting in $T_{\mathrm{c}} \sim 6.5 \mathrm{~K}$. Together with the zero temperature gap value of $\Delta_{0}=1.05 \mathrm{meV}$ extrapolated from the BCS fit we deduce a ratio of $2 \Delta_{0} / k_{\mathrm{B}} T_{\mathrm{c}} \sim 3.8$ for one UC thin FeTe on $\mathrm{Bi}_{2} \mathrm{Te}_{3}$, which is comparable to the value of 4.2 we determine for the $\mathrm{FeTe}_{0.5} \mathrm{Se}_{0.5}$ thin film on $\mathrm{Bi}_{2} \mathrm{Te}_{1.8} \mathrm{Se}_{1.2}$, but 
a

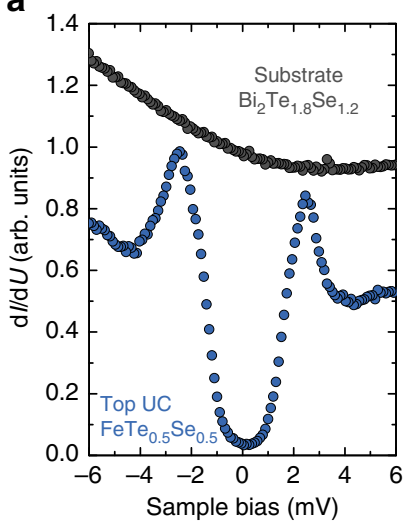

d

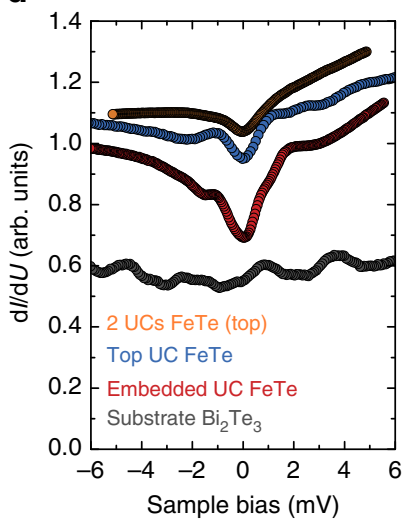

b

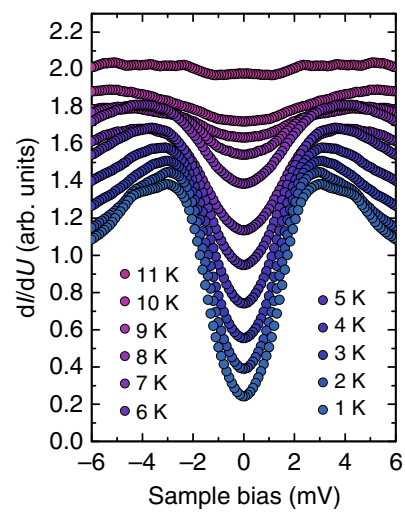

e

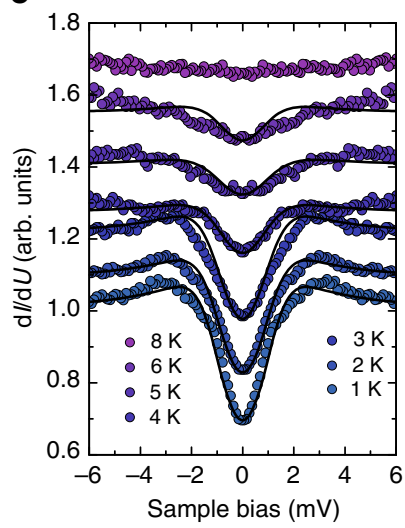

c

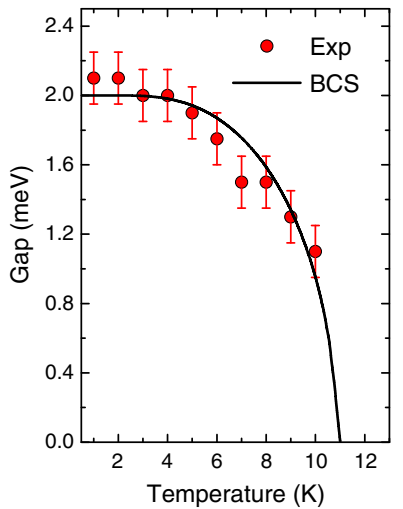

f

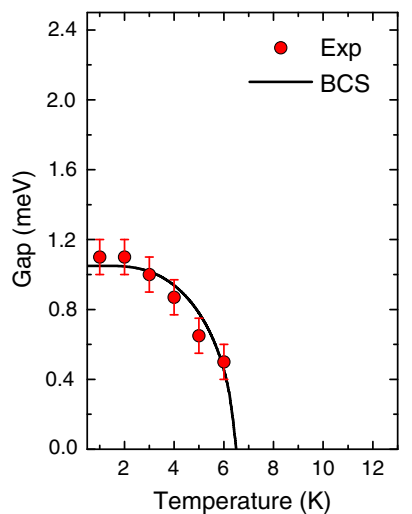

Figure 2 | Thickness and temperature dependence of STS on $\mathbf{F e T e}_{\mathbf{1}} \mathbf{S e}_{\mathbf{x}}$ (a) $\mathrm{d} / \mathrm{dU}$ spectra of one $U \mathrm{C}$ thin $\mathrm{FeTe}_{0.5} \mathrm{Se}_{0.5}$ and of $\mathrm{Bi}_{2} \mathrm{Te}_{1.8} \mathrm{Se}_{1.2}$ substrate measured at $T=1.1 \mathrm{~K}\left(U=10 \mathrm{mV}, I_{\mathrm{s}}=400 \mathrm{pA}, U_{\bmod }=0.1 \mathrm{mV}\right)$. (b) Temperature evolution of the $\mathrm{d} / / \mathrm{dU}$ spectra on a top $U C$ thin FeTe $\mathrm{F}_{0.5} \mathrm{Se}_{0.5}(U=10 \mathrm{mV}$, $I_{\mathrm{s}}=200 \mathrm{pA}, U_{\text {mod }}=0.15 \mathrm{mV}$, spectra vertically offset for clarity). The spectra are spatially averaged along a line of length $1 \mathrm{~nm}$, background-corrected by division through the data measured above $T_{\mathrm{c}}\left(\right.$ at $T=14 \mathrm{~K}$ ) and finally symmetrized with respect to $U=0 \mathrm{~V}$. (c) Energy gaps $\Delta$ of a top $U C$ thin FeTe ${ }_{0.5} \mathrm{Se}_{0.5}$ as a function of temperature. Markers show the gaps as extracted from Dynes fits (not shown) to the data in $\mathbf{b}$, with error bars indicating the maximum range of values that result in an acceptable fitting of the experimental spectra, and the solid line shows the fitting to the BCS gap function. (d) Typical spatially averaged $\mathrm{d} / \mathrm{d} U$ spectra measured on ultrathin FeTe films of different thicknesses and on the $\mathrm{Bi}_{2} \mathrm{Te}_{3}$ substrate at $T=1.1 \mathrm{~K}$. The spectra have been normalized to the $\mathrm{d} / \mathrm{d} U$ value at $+5 \mathrm{mV}$ and are vertically offset (substrate: offset $-0.4, U=10 \mathrm{mV}, I_{\mathrm{s}}=170 \mathrm{pA}, U_{\text {mod }}=0.2 \mathrm{mV}$; embedded $U \mathrm{C}$ : offset $+0.1, U=6 \mathrm{mV}, I_{\mathrm{s}}=300 \mathrm{pA}, U_{\text {mod }}=0.2 \mathrm{mV}$; top UC: offset $+0.2, U=10 \mathrm{mV}, I_{\mathrm{s}}=600 \mathrm{pA}, U_{\text {mod }}=0.25 \mathrm{mV} ; 2 \mathrm{UCs}$ : offset $+0.3, U=5 \mathrm{mV}, I_{\mathrm{s}}=300 \mathrm{pA}$, $\left.U_{\text {mod }}=0.2 \mathrm{mV}\right)$. (e) Spatially averaged $(0.5 \mathrm{~nm} \times 0.5 \mathrm{~nm}) \mathrm{dl} / \mathrm{dU}$ spectra measured of a top $U C$ thin FeTe layer at temperatures from 1.1 to $8 \mathrm{~K}(U=10 \mathrm{mV}$, $I_{\mathrm{s}}=200 \mathrm{pA}, U_{\text {mod }}=0.15 \mathrm{mV}$, spectra vertically offset for clarity). The spectra are background-corrected by division through the data measured at $T=8 \mathrm{~K}$, and finally symmetrized with respect to $U=0 \mathrm{~V}$. The lines are fits to Dynes functions. (f) Energy gaps $\Delta$ of top UC FeTe, as obtained from the fits in $\mathbf{e}$, as a function of temperature. The errors indicate the maximum range of values of $\Delta$ in the Dynes fits, which result in an acceptable agreement with the experimental spectra. The solid line represents the temperature variation of $\Delta$ as fitted by BCS theory.

somewhat larger than typical values of $2.5-3.2$ for bulk $\mathrm{FeTe}_{1-x} \mathrm{Se}_{x}$ samples ${ }^{23,26}$. We note that the embedded UC of FeTe also shows a similar temperature-dependent behaviour (Supplementary Fig. 7). Our results are consistent with the transport measurements of $\mathrm{Fe}_{1+x} \mathrm{Te}$ films grown on $\mathrm{Bi}_{2} \mathrm{Te}_{3}$ (ref. 15) where evidence for superconductivity with a $T_{\mathrm{c}} \sim 12 \mathrm{~K}$ was reported. We do not observe any significant effect on the energy gap in a magnetic field up to $B=2.8 \mathrm{~T}$ applied perpendicular to the sample plane (Supplementary Fig. 8). This suggests that the robustness of the pairing with respect to an external magnetic field is similarly strong as for other Fe-based superconducting thin films ${ }^{7}$, and an identical effect has been observed in magnetotransport measurements of a $\mathrm{Bi}_{2} \mathrm{Te}_{3} / \mathrm{FeTe}$ heterostructure ${ }^{14,15}$.

Spatial variation of superconducting correlation. Having established clear evidence for superconducting correlations in single UC FeTe layers grown on $\mathrm{Bi}_{2} \mathrm{Te}_{3}$ below $6 \mathrm{~K}$, we now focus in more detail on the spatial behaviour of these correlations at the lateral interface of the $\mathrm{FeTe}$ layer and the $\mathrm{Bi}_{2} \mathrm{Te}_{3}$ substrate. In particular, we measured the spatial variation of tunnelling spectra across a step edge from the embedded UC FeTe layer to the $\mathrm{Bi}_{2} \mathrm{Te}_{3}$ substrate (Fig. 3a,b). The resulting tunnelling spectra across the interface along the line shown in Fig. 3 a obtained at $T=1.1 \mathrm{~K}$ (Fig. 3c) again show the presence of the gap due to superconducting correlations on top of the FeTe layer, which vanishes on the TI substrate. To quantify the decay length of the superconducting correlations in our heterostructure system, we have fitted the spatial dependence of the gap area with an exponential decay function (Fig. 3d), resulting in a decay length of about $\xi=8.9 \AA$ at $T=1.1 \mathrm{~K}$. This decay length is a measure for the coherence length of the Cooper pairs ${ }^{31,32}$, which is obviously similarly small as, for example, in bulk $\mathrm{FeTe}_{0.6} \mathrm{Se}_{0.4}$ (ref. 26).

Magnetic properties using SP-STM. Our results on epitaxially grown FeTe layers on $\mathrm{Bi}_{2} \mathrm{Te}_{3}$ confirm that the previously reported 
a

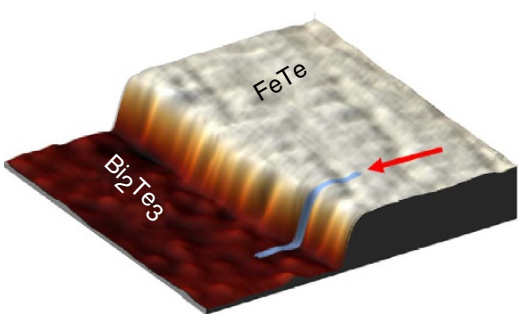

b

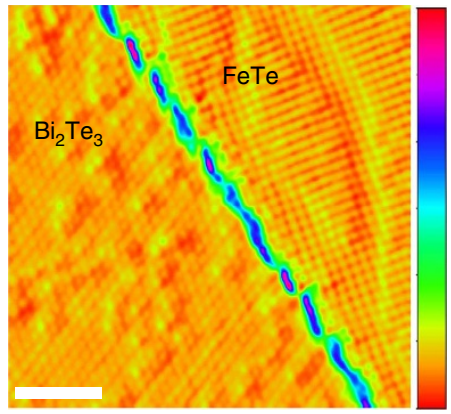

C
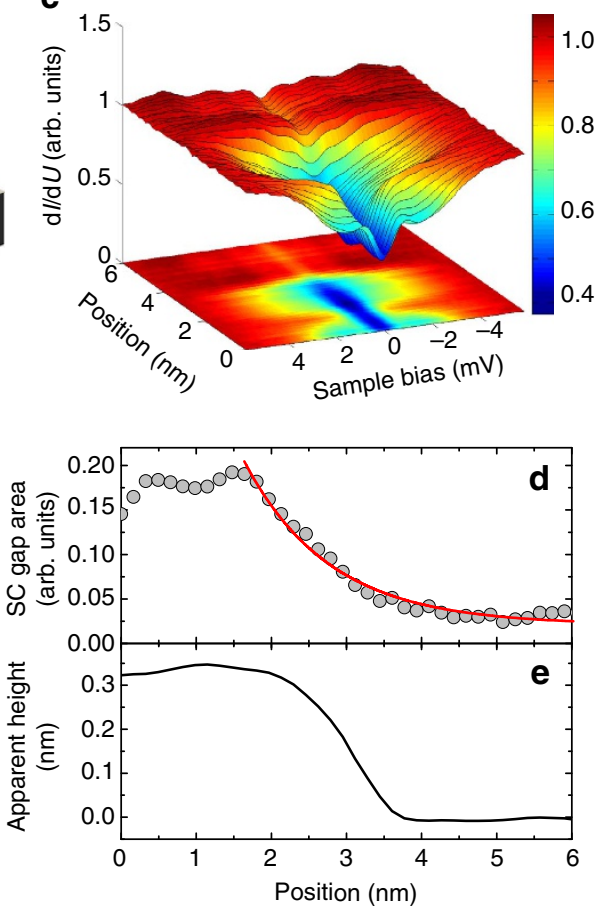

Figure 3 | Spatial variation of energy gap across a lateral interface. (a) STM topography ( $25 \mathrm{~nm} \times 25 \mathrm{~nm}$ ) showing a $\mathrm{Bi}_{2} \mathrm{Te}_{3}$ terrace and an embedded UC thin FeTe layer grown on top $\left(U=100 \mathrm{mV}, I_{\mathrm{s}}=50 \mathrm{pA}\right)$. (b) Atomically resolved STM topography of a similar area $\left(U=50 \mathrm{mV}, I_{\mathrm{s}}=50 \mathrm{pA}\right.$, differentiated with respect to horizontal axis, white scale bar, $3 \mathrm{~nm}$ wide). (c) Two- and three-dimensional representation of normalized and symmetrized $\mathrm{d} / / \mathrm{d} U \mathrm{spectra}$ taken across the step along the line indicated in $\mathbf{a}$ in the direction of the arrow. (d) Evolution of the gap (markers), determined from the spectra in $\mathbf{c}$ by measuring the area enclosed by the gap within a $\pm 1 \mathrm{meV}$ voltage window. The solid line shows a fit to an exponential decay resulting in a decay length of $\xi=8.9 \AA$. (e) Topographic height taken along the same line used for the data in $\mathbf{c}, \mathbf{d}$

two-dimensional superconductivity in $\mathrm{FeTe} / \mathrm{Bi}_{2} \mathrm{Te}_{3}$ heterostructures $^{14,15}$ is associated with the presence of a superconducting layer of FeTe located at the interface. We now return to the central question of whether the usual bi-collinear AFM order of bulk FeTe (ref. 16) is suppressed in the FeTe layer interfacing $\mathrm{Bi}_{2} \mathrm{Te}_{3}$, as proposed by $\mathrm{He}$ et al. ${ }^{14}$, or whether it can coexists with the superconducting correlations we observed.

In order to check this assumption, we simultaneously characterized the local superconducting correlations and the atomic-scale spin structure of the ultrathin FeTe films grown on $\mathrm{Bi}_{2} \mathrm{Te}_{3}$ by SP$\mathrm{STS}^{33}$. By using a spin-polarized bulk $\mathrm{Cr}$ tip $^{34}$, we excluded the disturbing influence of a local magnetic stray field on the sample while being sensitive to its out-of-plane surface spin component. Figure $4 \mathrm{a}$ represents the spin-resolved constant-current image of a single UC thin embedded FeTe layer on $\mathrm{Bi}_{2} \mathrm{Te}_{3}$ as obtained in an external magnetic field of $B=+1 \mathrm{~T}$ applied perpendicular to the sample surface. The SP-STM image shows a clear stripe-like pattern, superimposed on the square atomic lattice of surface Te atoms, which is significantly different from the STM topography obtained with a non-magnetic PtIr tip as presented in Fig. If. The Fourier transform (inset of Fig. 4a) of the SP-STM image reveals an additional pair of peaks along the $a$ direction with a wave vector $\mathbf{q}_{\mathrm{AFM}}=1 / 2 \mathbf{q}_{\mathrm{Te}}^{a}$ that is half as long as that of the atomic lattice Bragg peaks. This unidirectional modulation with a characteristic periodicity of $\lambda=2 a=7.5 \AA$ (see Fig. $4 \mathrm{e}$ ) has been attributed to a direct observation of bi-collinear AFM order of the Fe atoms below the surface of bulk $\mathrm{Fe}_{1+\mathrm{y}} \mathrm{Te}$ using SP-STM ${ }^{16}$. To confirm the magnetic origin of the observed superstructure with twice the atomic lattice periodicity, we performed an SP-STM measurement at the same location with an oppositely aligned effective spin at the Cr tip's front end by reversing the magnetic field to $B=-1 \mathrm{~T}$ (see Fig. $4 \mathrm{~b}$ ). Now, the $2 a$ superstructure reveals a phase shift (indicated by an arrow) of one atomic lattice unit as expected for a magnetic origin of the contrast. The maximum spin contrast appears between every second Fe lattice site along a diagonal of the surface UC being located between two neighbouring Te sites. This can be explained by the fact that spin-polarized tunnelling primarily results from the $3 d$ states of Fe being located below the top Te layer. It is also clearly visible from the line profiles of Fig. $4 \mathrm{e}$ along the $a$ direction, which were extracted from Fig. $4 \mathrm{a}, \mathrm{b}$, and by the $2 a$ periodicity along the $a$ direction in the out-of-plane spinpolarization distribution of the FeTe layer (Fig. 4c) obtained by subtracting the SP-STM images of Fig. 4a,b. These observations prove that single UC FeTe films on $\mathrm{Bi}_{2} \mathrm{Te}_{3}$ exhibit a bi-collinear AFM structure, similar to the one known for bulk FeTe (ref. 16), but with a non-vanishing out-of-plane surface spin component ${ }^{35}$. Note that the same bi-collinear AFM structure was also observed for the thicker islands in our samples (Supplementary Fig. 9). To our knowledge, this is the first direct real-space observation of magnetic order in an Fe-chalcogenide compound at the single UC thickness limit.

The observed coexistence of magnetic order in UC FeTe, which is similar to the one found at the surface of the corresponding bulk material ${ }^{16}$, and superconducting correlations, which are not present in bulk FeTe, is quite surprising. In order to find a mutual relationship between magnetic order and superconductivity, we performed SP-STM experiments as a function of temperature from 1 to $10 \mathrm{~K}$. Representative SP-STM images and corresponding FFT data obtained at temperatures $T=1,6$ and $10 \mathrm{~K}$ are shown in Fig. 5a-f. The position of the surface area is the same in all three SP-STM data sets. The spin contrast is clearly reflected by the superstructure with twice the atomic lattice periodicity, and in the corresponding FFT data by the spots at wave vector $\mathbf{q}_{\mathrm{AFM}}=1 / 2$ $\mathbf{q}_{\text {Te }}^{a}$. Most notably, the spin structure does not change and the 

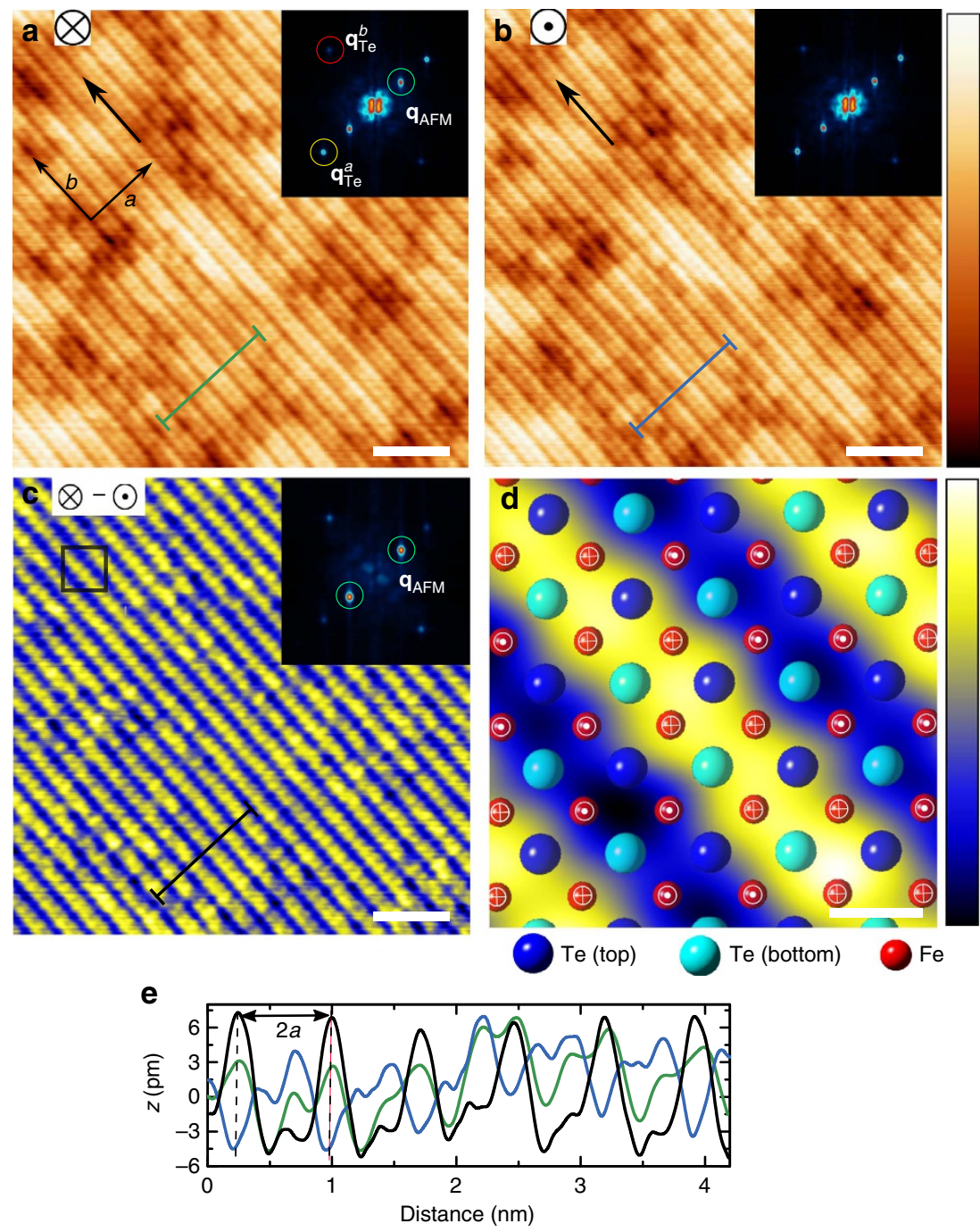

Figure 4 | Bi-collinear AFM order in embedded and top UC FeTe. (a) SP-STM image recorded at $B=+1$ showing spin order of one UC FeTe at $T=1.1 \mathrm{~K}$ using an out-of-plane spin-sensitive bulk $\mathrm{Cr}$ tip. (b) SP-STM image of the same area acquired at $B=-1 \mathrm{~T}$ with an oppositely spin-oriented tip ( $U=300 \mathrm{mV}$, $I_{\mathrm{s}}=220 \mathrm{pA}$, white scale bars, $3 \mathrm{~nm}$ wide, colour scale from 0 to $33 \mathrm{pm}$ apparent height). The arrows in $\mathbf{a}, \mathbf{b}$ indicate the same sample spot. The insets in $\mathbf{a}, \mathbf{b}$ show FFTs of the corresponding SP-STM images, where the peaks marked by yellow and red circles are associated with the top Te atomic square lattice (Bragg peaks) at $\mathbf{q}_{\mathrm{Te}}^{a}$ and $\mathbf{q}_{\mathrm{T}}^{b}$ (image sizes of all FFTs is $0.55 \AA^{-1}$ ). Besides the Te Bragg peaks, the FFTs reveal pairs of additional strong peaks (green circles) associated with AFM order at $\mathbf{q}_{\mathrm{AFM}}=1 / 2 \mathbf{q}_{\mathrm{Te}}^{a}$. (c) Spin asymmetry map obtained by subtraction of images a,b, revealing bi-collinear AFM order in the one UC thin FeTe layer (white scale bar, $3 \mathrm{~nm}$ wide). Inset: FFT pattern of the difference image highlighting the peaks in reciprocal space resulting from AFM spin order. (d) Model representation of the atomic structure and of the out-of-plane components of magnetic moments superimposed on the zoomed-in spin asymmetry image of $\mathbf{c}$ (white scale bar, $2.8 \AA$ wide, colour scale from -12 to $12 \mathrm{pm}$ ). (e) Measured profiles along identical lines drawn in images a-c, showing the periodicity of AFM order in real space, that is, $\lambda=2 a$.

intensity of the spin contrast is almost the same for all three temperatures shown here. Similar SP-STM images at other temperatures have been taken in order to quantify the temperature evolution of the spin contrast via the ratio of FFT intensities of the $\mathbf{q}_{\mathrm{AFM}}$ spots to the Bragg spots at $\mathbf{q}_{\mathrm{T}}^{a}$. Figure $5 \mathrm{~g}$ shows this evolution together with the evolution of the superconducting energy gap taken from Fig. 2f. The comparison clearly demonstrates that the bi-collinear AFM order is largely unaffected by the disappearance of the superconducting correlations. Therefore, our experimental results do not provide any evidence that one kind of order emerges at the expense of the other, nor does the data provide any indication for a microscopic phase separation into regions with superconducting and magnetic order. Our findings for single UC thin FeTe layers on $\mathrm{Bi}_{2} \mathrm{Te}_{3}$ therefore challenge the common belief that optimal superconducting pairing sets in when long-range AFM order is suppressed in the parent compound.

\section{Discussion}

In summary, we have explored the electronic and magnetic structure in a new class of systems, that is, heterostructures consisting of ultrathin Fe-chalcogenide layers of the type $\mathrm{FeTe}_{1-}$ ${ }_{x} \mathrm{Se}_{x}(x=0,0.5)$ on Bi-based TI substrates. We observe fully developed U-shaped superconducting gaps in $\mathrm{FeTe}_{0.5} \mathrm{Se}_{0.5}$ layers of one UC thickness with a transition temperature of $T_{c} \sim 11 \mathrm{~K}$, close to the one of the corresponding bulk system $\left(T_{\mathrm{c}} \sim 14.5 \mathrm{~K}\right)$. For $\mathrm{FeTe} / \mathrm{Bi}_{2} \mathrm{Te}_{3}$ heterostructures, our atomic-scale spin-resolved tunnelling spectroscopy measurements provide evidence for the coexistence of superconducting correlations with $T_{\mathrm{c}} \sim 6.5 \mathrm{~K}$ and bi-collinear AFM order in the one UC FeTe films. Although the coexistence of static magnetic order and superconductivity was observed earlier by spatially averaging techniques in several systems such as Fe-pnictides 36,37 , heavy fermion compounds ${ }^{38}$ and Fe-chalcogenides ${ }^{39}$, the exact microscopic picture of the 

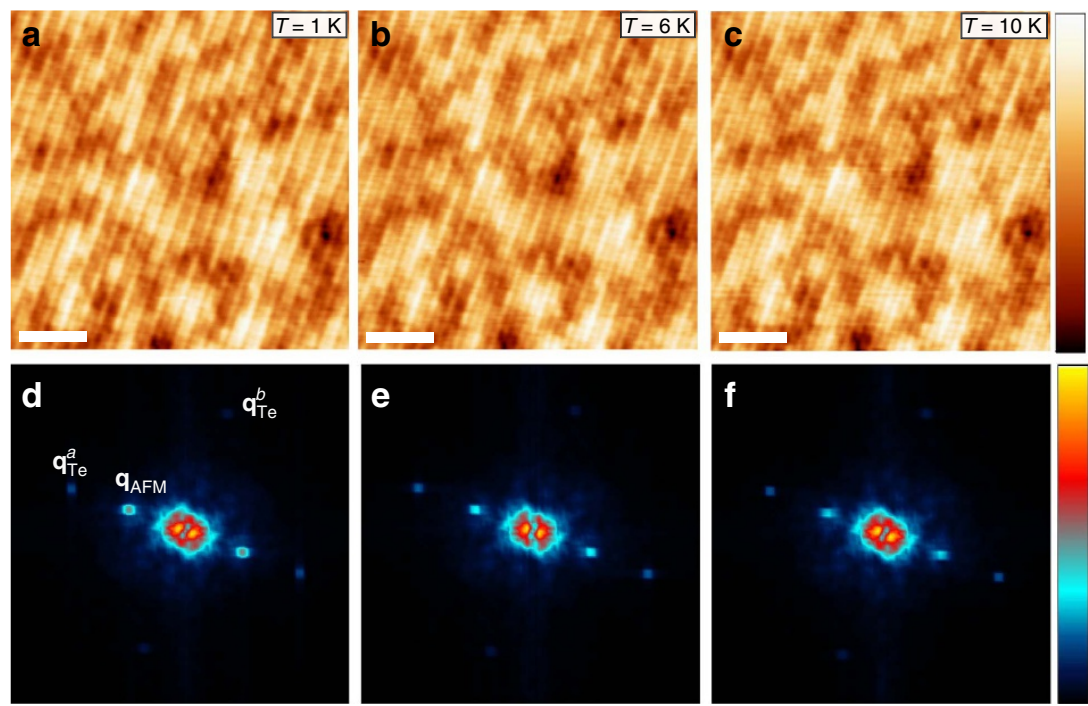

g

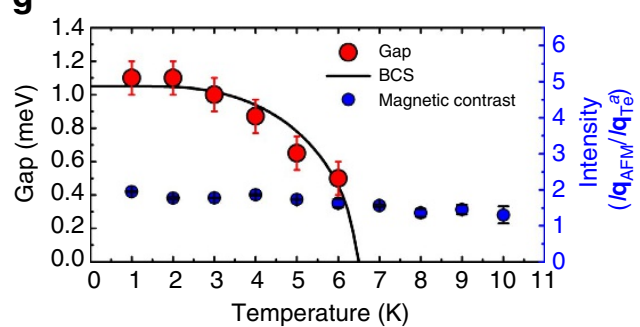

Figure 5 | Temperature stability of spin structure for top UC FeTe. (a-c) SP-STM images $\left(U=100 \mathrm{mV}\right.$ and $I_{\mathrm{s}}=100 \mathrm{pA}$, white scale bars, $3 \mathrm{~nm}$ wide, colour scale from 0 to $31 \mathrm{pm}$ apparent height) and (d-f) corresponding FFT data (image size $0.75 \AA^{-1}$ ) acquired at $B=0.5$ T using an out-of-plane sensitive $\mathrm{Cr}$ tip, taken at different temperatures as indicated in a-c. The position of the surface area is the same in all three SP-STM data sets. The spin structure is robust and the intensity of the magnetic contrast is almost the same at all three temperatures. (g) Temperature dependence of the energy gaps $\Delta$ plotted together with the magnetic contrast quantified via the ratio of intensities of the FFT spots at $\mathbf{q}_{\mathrm{AFM}}$ and $\mathbf{q}_{\text {Te }}^{a}$ from the SP-STM images in $\mathbf{d}-\mathbf{f}$ and similar images taken at other temperatures. The errors in $\Delta$ indicate the maximum range of values used in the Dynes fits, which result in an acceptable agreement with the experimental spectra. The errors in the magnetic contrast indicate the mean deviation as estimated from two data sets.

coexistence still remained unclear because of a lack of spatially resolved data ${ }^{40}$. Here we can compare the wavelength of the AFM order of $\lambda=2 a=7.5 \AA$ with the size of the Cooper pairs, inferred from the coherence length, of $\xi=8.9 \AA$, giving $\xi / \lambda \sim 1.2$. The electron distance in the pairs is rather small, which is typical for Fe-based SCs, and apparently just large enough such that the effective Zeeman field induced by the AFM order cancels out along the length of the Cooper pair. The relative sizes of $\lambda$ and $\xi$ therefore might be crucial for the coexistence of pairing and longrange AFM order in this material. Our surprising findings may stimulate further theoretical studies on the relationship between superconductivity and AFM order in Fe-based SCs.

We finally note that the leakage of the gap into the TI substrate across the $\mathrm{FeTe}-\mathrm{Bi}_{2} \mathrm{Te}_{3}$ interface (Fig. 3c) indicates the presence of superconducting correlations in the TI material close to the interface. The atomic sharpness of this interface suggests that the topological surface state of the TI substrate stays intact as also shown recently by photoemission experiments for the case of a FeSe- $-\mathrm{Bi}_{2} \mathrm{Se}_{3}$ heterostructure ${ }^{41}$. The $\mathrm{FeTe}-\mathrm{Bi}_{2} \mathrm{Te}_{3}$ interface therefore provides an ideal platform to study the interesting physics of Dirac fermions interacting with Cooper pairs.

\section{Methods}

Samples. Bulk TI single crystals of $\mathrm{Bi}_{2} \mathrm{Te}_{3}$ and $\mathrm{Bi}_{2} \mathrm{Te}_{1.8} \mathrm{Se}_{1.2}$ used in this study as substrates were synthesized using a Stockbarger method and have been well characterized using angle-resolved photoemission spectroscopy, powder X-ray diffraction, inductively coupled plasma atomic-emission spectroscopy and potential Seebeck microprobe measurements ${ }^{21,42}$. For all substrates the Dirac point is located energetically below the Fermi level indicating $n$ doping. Fe-chalcogenide film preparation and characterization were carried out in a UHV system with a base pressure below $1 \times 10^{-10}$ mbar. The TI crystals were cleaved in situ under UHV conditions and high-quality $\mathrm{FeTe}_{1-x} \mathrm{Se}_{x}(x=0,0.5)$ thin films were prepared by depositing 0.5-1 ML Fe at $300 \mathrm{~K}$ on-top of $\mathrm{Bi}_{2} \mathrm{Te}_{3}$ and $\mathrm{Bi}_{2} \mathrm{Te}_{1.8} \mathrm{Se}_{1.2}$ substrates, respectively, using molecular beam epitaxy, followed by annealing up to a maximum temperature of $315^{\circ} \mathrm{C}$ for $15 \mathrm{~min}$. Fe deposited on $\mathrm{Bi}_{2} \mathrm{Te}_{3}$ reacts with the substrate upon annealing, most likely via a substitutional process of $\mathrm{Bi}$ by $\mathrm{Fe}$ (ref. 43), leading to a high-quality defect-free FeTe film.

Experimental techniques. All STM/STS experiments were performed with an STM in UHV at temperatures between 1.1 and $14 \mathrm{~K}$ (ref. 24). A magnetic field $B$ of up to $3 \mathrm{~T}$ can be applied perpendicular to the sample surface. Topography images were obtained in constant-current mode with stabilization current $I_{\mathrm{s}}$ and bias voltage $U$ applied to the sample. STS data were obtained using a lock-in technique to record the differential tunnelling conductance $\mathrm{d} / \mathrm{d} U$ by adding an AC modulation voltage $U_{\text {mod }}$ (given in r.m.s.) to the bias voltage, after stabilizing the tip at $I_{\mathrm{s}}$ and $U$, switching off the feedback, and ramping the applied bias $U$. We used cut PtIr or electrochemically etched W tips (both in situ flashed) for spin-averaged imaging and spectroscopy measurements. For spin-resolved measurements, we used bulk $\mathrm{Cr}$ tips, which were prepared by electrochemical etching followed by a high-voltage field emission treatment using $\mathrm{W}(110)$ or $\mathrm{Ta}(001)$ as a substrate.

Data availability. The authors declare that the main data supporting the findings of this study are available within the article and its Supplementary Information files. Extra data are available from the corresponding author upon request.

\section{References}

1. Bozovic, I. \& Ahn, C. A new frontier for superconductivity. Nat. Phys. 10, 892-895 (2014). 
2. Qi, X.-L. \& Zhang, S.-C. Topological insulators and superconductors. Rev. Mod. Phys. 83, 1057-1110 (2011).

3. Fu, L. \& Kane, C. L. Superconducting proximity effect and Majorana Fermions at the surface of a topological insulator. Phys. Rev. Lett. 100, 096407 (2008).

4. Xu, J. P. et al. Experimental detection of a Majorana mode in the core of a magnetic vortex inside a topological insulator-superconductor $\mathrm{Bi}_{2} \mathrm{Te}_{3} / \mathrm{NbSe}_{2}$ heterostructure. Phys. Rev. Lett. 114, 017001 (2015).

5. Paglione, J. \& Greene, R. L. High-temperature superconductivity in iron-based materials. Nat. Phys. 6, 645-658 (2010).

6. Stewart, G. R. Superconductivity in iron compounds. Rev. Mod. Phys. 83, 1589-1652 (2011)

7. Wang, Q. Y. et al. Interface-induced high-temperature superconductivity in single unit-cell FeSe films on $\mathrm{SrTiO}_{3}$. Chin. Phys. Lett. 29, 037402 (2012).

8. Ge, G. F. et al. Superconductivity above $100 \mathrm{~K}$ in single-layer FeSe films on doped $\mathrm{SrTiO}_{3}$. Nat. Mater. 14, 285-289 (2015).

9. Sun, Y. et al. High temperature superconducting FeSe films on $\mathrm{SrTiO}_{3}$ substrates. Sci. Rep. 4, 06040 (2014).

10. Hsu, F. C. et al. Superconductivity in the PbO-type structure $\alpha$-FeSe. Proc. Natl Acad. Sci. USA 105, 14262-14264 (2008).

11. Peng, R. et al. Tuning the band structure and superconductivity in single-layer FeSe by interface engineering. Nat. Commun. 5, 5044 (2014).

12. Li, F. et al. Interface-enhanced high-temperature superconductivity in singleunit-cell $\mathrm{FeTe}_{1-x} \mathrm{Se}_{x}$ films on $\mathrm{SrTiO}_{3}$. Phys. Rev. B 91, 220503(R) (2015).

13. Bert, J. A. et al. Direct imaging of the coexistence of ferromagnetism and superconductivity at the $\mathrm{LaAlO}_{3} / \mathrm{SrTiO}_{3}$ interface. Nat. Phys. 7, 767-771 (2011).

14. He, Q. L. et al. Two-dimensional superconductivity at the interface of a $\mathrm{Bi}_{2} \mathrm{Te}_{3} / \mathrm{FeTe}$ heterostructure. Nat. Commun. 5, 4247 (2014).

15. He, H. T. et al. Spontaneous vortex dynamics in superconducting FeTe thin films. Solid State Commun. 195, 35-38 (2014).

16. Enayat, M. et al. Real-space imaging of the atomic-scale magnetic structure of $\mathrm{Fe}_{1+y}$ Te. Science 345, 653-656 (2014)

17. Bao, W. et al. Tunable $(\delta \pi, \delta \pi)$-type antiferromagnetic order in $\alpha$-Fe(Te, Se) superconductors. Phys. Rev. Lett. 102, 247001 (2009).

18. Koz, C., Rößler, S., Tsirlin, A. A., Wirth, S. \& Schwarz, U. Low-temperature phase diagram of $\mathrm{Fe}_{1+y}$ Te studied using x-ray diffraction. Phys. Rev. B 88, 094509 (2013)

19. Cavallin, A. et al. Preparation and characterization of $\mathrm{Bi}_{2} \mathrm{Se}_{3}(0001)$ and of epitaxial FeSe nanocrystals on $\mathrm{Bi}_{2} \mathrm{Se}_{3}(0001)$. Surf. Sci. 646, 72-82 (2016).

20. Kamlapure, A. et al. Evidence for a two-fold symmetric superconducting gap in a monolayer of $\mathrm{FeSe}_{0.5} \mathrm{Te}_{0.5}$ on a topological insulator. Preprint at http:// arxiv.org/abs/1608.03827 (2016).

21. Mi, J.-L. et al. Phase separation and bulk p-n transition in single crystals of $\mathrm{Bi}_{2} \mathrm{Te}_{2} \mathrm{Se}$ topological insulator. Adv. Mater. 25, 889-893 (2013).

22. He, X. et al. Nanoscale chemical phase separation in $\mathrm{FeTe}_{0.55} \mathrm{Se}_{0.45}$ as seen via scanning tunneling spectroscopy. Phys. Rev. B 83, 220502(R) (2011).

23. Hanaguri, T., Niitaka, S., Kuroki, K. \& Takagi, H. Unconventional s-wave superconductivity in $\mathrm{Fe}(\mathrm{Se}, \mathrm{Te})$. Science 328, 474-476 (2010).

24. Löptien, P., Zhou, L., Khajetoorians, A. A., Wiebe, J. \& Wiesendanger, R. Superconductivity of lanthanum revisited: enhanced critical temperature in the clean limit. J. Phys. Condens. Matter 26, 425703 (2014).

25. Tinkham, M. Introduction to Superconductivity 2nd edn, Vol i(Dover Books on Physics, 2004).

26. Singh, U. R. et al. Spatial inhomogeneity of the superconducting gap and order parameter in $\mathrm{FeSe}_{0.4} \mathrm{Te}_{0.6}$. Phys. Rev. B 88, 155124 (2013).

27. Sugimoto, A., Ekino, T. \& Gabovich, A. M. Variable electronic stripe structures of the parent iron-chalcogenide superconductor $\mathrm{Fe}_{1+d} \mathrm{Te}$ observed by STM-STS. Phys. Rev. B 90, 224503 (2014).

28. Li, W. et al. Charge ordering in stoichiometric FeTe: scanning tunneling microscopy and spectroscopy. Phys. Rev. B 93, 041101(R) (2016).

29. Ernst, S. et al. Emerging local Kondo screening and spatial coherence in the heavy-fermion metal $\mathrm{YbRh}_{2} \mathrm{Si}_{2}$. Nature 474, 362-366 (2011).

30. Fischer, Ø., Kugler, M., Maggio-Aprile, I. \& Berthod, C. Scanning tunneling spectroscopy of high-temperature superconductors. Rev. Mod. Phys. 79, 353-419 (2007).

31. Kim, J. et al. Visualization of geometric influences on proximity effects in heterogeneous superconductor thin films. Nat. Phys. 8, 464-469 (2012).

32. Caminale, M. et al. Threshold of magnetic field response of the superconducting proximity effect for ultrathin $\mathrm{Pb} / \mathrm{Ag}$ metallic films. Phys. Rev. B 90, 220507(R) (2014).
33. Wiesendanger, R. Spin mapping at the nanoscale and atomic scale. Rev. Mod. Phys. 81, 1495-1550 (2009).

34. Schlenhoff, A., Krause, S., Herzog, G. \& Wiesendanger, R. Bulk Cr tips with full spatial magnetic sensitivity for spin-polarized scanning tunneling microscopy. Appl. Phys. Lett. 97, 083104 (2010).

35. Hänke, T. et al. Reorientation of the diagonal double-stripe spin structure at $\mathrm{Fe}_{1+y} \mathrm{Te}$ bulk and thin-film surfaces. Nat. Commun 8, 13939 (2017).

36. Drew, A. J. et al. Coexistence of static magnetism and superconductivity in $\mathrm{SmFeAsO} \mathrm{H}_{1-x} \mathrm{~F}_{x}$ as revealed by muon spin rotation. Nat. Mater. 8, 310-314 (2009).

37. Dai, P. Antiferromagnetic order and spin dynamics in iron-based superconductors. Rev. Mod. Phys. 87, 855-896 (2015).

38. Kratochvílová, M. et al. Coexistence of antiferromagnetism and superconductivity in heavy Fermion cerium compound $\mathrm{Ce}_{3} \mathrm{PdIn}_{11}$. Sci. Rep. 5, 15904 (2015).

39. Khasanov, R. et al. Coexistence of incommensurate magnetism and superconductivity in $\mathrm{Fe}_{1+y} \mathrm{Se}_{x} \mathrm{Te}_{1-x}$. Phys. Rev. B 80, 140511(R) (2009).

40. Choi, S. et al. Atomic scale observation and manipulation of plaquette antiferromagntic order coexisting with iron superconductivity. Preprint at http://arxiv.org/abs/1608.00884 (2016).

41. Eich, A. et al. Absence of superconductivity in ultra-thin layers of FeSe synthesized on a topological insulator. Phys. Rev. B 94, 125437 (2016).

42. Vondráček, M. et al. Nickel: the time-reversal symmetry conserving partner of iron on a chalcogenide topological insulator. Phys. Rev. B 94, 161114 (2016).

43. Schlenk, T. et al. Controllable magnetic doping of the surface state of a topological insulator. Phys. Rev. Lett. 110, 126804 (2013).

\section{Acknowledgements}

We are indebted to Alexander Balatsky, Christopher Triola, Tim O. Wehling and Udai R. Singh for valuable discussions. This work has primarily been supported by the ERC Advanced Grant ASTONISH (No. 338802). L.C., Ph.H. and J.W. acknowledge support through the DFG priority programme SPP1666 (grant no. WI 3097/2), and T.H. acknowledges support by the DFG under grant No. HA 6037/2-1. We thank the Aarhus University Research Foundation for supporting the bulk crystal growth.

\section{Author contributions}

S.M., J.W., T.H. and R.W. designed the experiment. S.M., A.K. and L.C. have grown and characterized the thin-film samples, performed the STM/STS and SP-STS experiments and analysed the data. S.M., R.W. and J.W. wrote the manuscript. E.M.J.H., M.B., B.B.I and Ph.H. have grown and characterized the TI single crystals. All authors discussed the results and contributed to the manuscript.

\section{Additional information}

Supplementary Information accompanies this paper at http://www.nature.com/ naturecommunications

Competing financial interests: The authors declare no competing financial interests.

Reprints and permission information is available online at http://npg.nature.com/ reprintsandpermissions/

How to cite this article: Manna, S. et al. Interfacial superconductivity in a bi-collinear antiferromagnetically ordered FeTe monolayer on a topological insulator. Nat. Commun. 8, 14074 doi: $10.1038 /$ ncomms14074 (2017).

Publisher's note: Springer Nature remains neutral with regard to jurisdictional claims in published maps and institutional affiliations.

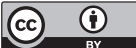

This work is licensed under a Creative Commons Attribution 4.0 International License. The images or other third party material in this article are included in the article's Creative Commons license, unless indicated otherwise in the credit line; if the material is not included under the Creative Commons license, users will need to obtain permission from the license holder to reproduce the material. To view a copy of this license, visit http://creativecommons.org/licenses/by/4.0/

C) The Author(s) 2017 